\title{
Long-term chest CT follow-up in COVID-19 Survivors: 102-361 days after onset
}

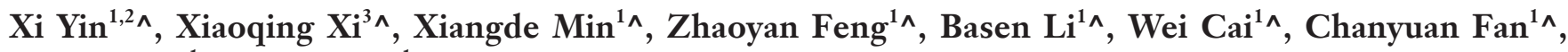 \\ Liang Wang ${ }^{1}$, Liming $\mathrm{Xia}^{1}{ }^{\wedge}$ \\ ${ }^{1}$ Department of Radiology, Tongji Hospital, Tongji Medical College, Huazhong University of Science and Technology, Wuhan, China; ${ }^{2}$ Department \\ of CT \& MRI, The First Affiliated Hospital, College of Medicine, Shihezi University, Shihezi, China; ${ }^{3}$ Department of Geriatrics, The First Affiliated \\ Hospital, College of Medicine, Shihezi University, Shihezi, China \\ Contributions: (I) Conception and design: X Yin, X Xi, X Min, L Wang, L Xia; (II) Administrative support: L Wang, L Xia; (III) Provision of study \\ materials or patients: X Yin, X Min, Z Feng, B Li, W Cai, C Fan; (IV) Collection and assembly of data: X Yin, X Xi, Z Feng, B Li, W Cai, C Fan; \\ (V) Data analysis and interpretation: X Yin, X Xi, X Min, Z Feng, B Li, W Cai, C Fan; (VI) Manuscript writing: All authors; (VII) Final approval of \\ manuscript: All authors. \\ Correspondence to: Liming Xia, MD, PhD. Department of Radiology, Tongji Hospital, Tongji Medical College, Huazhong University of Science and \\ Technology, 1095 Jiefang Anv., Wuhan 430030, China. Email: xialiming2017@outlook.com.
}

Background: The aim of this study was to evaluate long-term longitudinal changes in chest computed tomography (CT) findings in coronavirus disease 2019 (COVID-19) survivors and their correlations with dyspnea after discharge.

Methods: A total of 337 COVID-19 survivors who underwent CT scan during hospitalization and between 102 and 361 days after onset were retrospectively included. Subjective CT findings, lesion volume, therapeutic measures and laboratory parameters were collected. The severity of the survivors' dyspnea was determined by follow-up questionnaire. The evolution of the CT findings from the peak period to discharge and throughout follow-up and the abilities of CT findings and clinical parameters to predict survival with and without dyspnea were analyzed.

Results: Ninety-one COVID-19 survivors still had dyspnea at follow-up. The age, comorbidity score, duration of hospital stays, receipt of hormone administration, receipt of immunoglobulin injections, intensive care unit (ICU) admission, receipt of mechanical ventilation, laboratory parameters, clinical classifications and parameters associated with lesion volume of the survivors with dyspnea were significantly different from those of survivors without dyspnea. Among the clinical parameters and CT parameters used to identify dyspnea, parameters associated with lesion volume showed the largest area under the curve (AUC) values, with lesion volume at discharge showing the largest AUC (0.820). Lesion volume decreased gradually from the peak period to discharge and through follow-up, with a notable decrease observed after discharge. Absorption of lesions continued 6 months after discharge.

Conclusions: Among the clinical parameters and subjective CT findings, CT findings associated with lesion volume were the best predictors of post-discharge dyspnea in COVID-19 survivors.

Keywords: Coronavirus disease 2019 (COVID-19); computed tomography (CT); dyspnea; follow-up

Submitted Mar 25, 2021. Accepted for publication Jun 21, 2021.

doi: $10.21037 /$ atm-21-1438

View this article at: https://dx.doi.org/10.21037/atm-21-1438

^ ORCID: Xi Yin, 0000-0002-3004-3429; Xiaoqing Xi, 0000-0001-5108-3764; Xiangde Min, 0000-0002-1589-6861; Zhaoyan Feng, 0000-0002-

3568-2342; Basen Li, 0000-0001-5042-3552; Wei Cai, 0000-0003-1999-3498; Chanyuan Fan, 0000-0002-0131-2490; Liang Wang, 0000-0003-31412609; Liming Xia, 0000-0001-8481-3380. 


\section{Introduction}

The coronavirus disease 2019 (COVID-19) outbreak has lasted more than one year. According to official World Health Organization (WHO) statistics, as of 22 March 2021, the total number of confirmed cases of COVID-19 globally was over 122.82 million, and the total number of associated deaths was 2.71 million (1).

Up to $15 \%$ of COVID-19 patients require hospital admission, of whom $98.6 \%$ can be discharged from the hospital (2). However, discharge is not the end of the disease course, and in most patients, lung lesions are not completely absorbed at the time of discharge (3-6). Up to $98.1 \%$ of all chest computed tomography (CT) scans show abnormalities more than 28 days after symptom onset (7). Some studies have shown that many discharged patients experience longterm morbidities $(8,9)$. Therefore, it is of great significance to follow discharged COVID-19 patients.

Chest radiography is not advisable as a first-line technique for the detection of COVID-19 infection due to its limited ability to demonstrate ground-glass opacities (GGOs) (10). Point-of-care ultrasonography (POCUS) may help define the severity and progression of COVID-19 and is widely used in the evaluation of patients with dyspnea, especially in intensive care units (ICUs); however, POCUS may not provide findings as detailed or as early as CT (10). CT plays important roles in assessing the severity of COVID-19 disease and evaluating therapeutic effects $(11,12)$. Several studies have evaluated the CT findings of COVID-19 patients in short-term follow-up (6,13-16). In one study, the CT imaging findings of COVID-19 were divided into 4 stages according to the number of days since the onset of symptoms (17). The results suggested that the early CT findings in COVID-19 patients are complex, can change rapidly, and can predict the outcome at discharge. To date, there have been few reports on the long-term changes in CT findings in COVID-19 survivors after discharge $(18,19)$. Thus, additional longitudinal studies are needed to develop viable rehabilitation practice guidelines for survivors. This study aimed to evaluate longitudinal changes in CT findings in COVID-19 survivors from the peak period to discharge and through long-term follow-up and to determine the correlations of clinical parameters and multiperiod CT findings with dyspnea after discharge.

We present the following article in accordance with the STROBE reporting checklist (available at https://dx.doi. org/10.21037/atm-21-1438).

\section{Methods}

\section{Patient selection and clinical classification of severity}

The study was conducted in accordance with the Declaration of Helsinki (as revised in 2013). The study was approved by the ethics committee of Wuhan Tongji Hospital (No. TJ-IRB20210120), and individual consent for this retrospective analysis was waived. Using the Electronic Medical Record Systems and Picture Archiving and Communication Systems, we retrospectively collected data of COVID-19 patients who underwent CT examination during hospitalization between January 1 and March 31, 2020, and who underwent follow-up CT examination between 102 and 361 days after onset. All cases were confirmed by reverse transcription polymerase chain reaction (RT-PCR) using nasopharyngeal swab samples. Patients in which the following criteria were met during follow-up were excluded: readmission for infection by other pathogens or extensive lung lesions due to non-COVID-19 reasons, such as pulmonary embolism or multiple lung metastases.

The diagnosis of COVID-19, the clinical classification of severity and the discharge criteria were determined according to the New Coronavirus Pneumonia Diagnosis and Treatment Plan (trial version 7) developed by the National Health Committee of the People's Republic of China (20). The clinical classification included mild, moderate, severe and critical.

\section{Clinical information collection}

Data on comorbidities, including chronic obstructive pulmonary disease (COPD), asthma, hypertension, coronary heart disease, diabetes, stroke and malignant tumor, were collected for all survivors. Some survivors had multiple comorbidities; each comorbidity received a score of 1 , so the comorbidity score for each survivor ranged from 0 to 7 .

Data on the main clinical treatments of COVID-19 and laboratory parameters reflecting tissue damage and inflammatory response were also collected. Treatments included hormone administration (methylprednisolone sodium succinate), immunoglobulin injection, antiviral therapy (arbidol), ICU admission, and mechanical ventilation for acute respiratory distress syndrome. Selected laboratory parameters included peak C-reactive protein (CRP), peak lactate dehydrogenase (LDH), peak D2- 


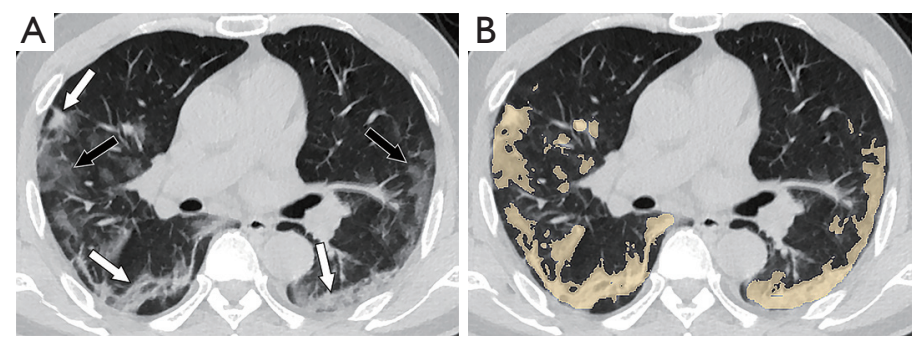

Figure 1 Thin-slice CT scan of a 57-year-old man with COVID-19 without dyspnea after discharge (obtained 9 days after onset) showing patchy consolidations (white arrows) and GGOs (black arrows) mainly in the bilateral subpleural region (A). 3D slicing was used to segment all lesions (B). CT, computed tomography; COVID-19, coronavirus disease 2019; GGOs, ground-glass opacities.

polymer, and peak calcitonin levels, peak neutrophil count, and minimum lymphocyte count.

The survivors were assessed for dyspnea with a followup questionnaire within one week after the follow-up CT scan. Dyspnea was scored from 1 to 4 according to the method reported by Staples et al. (21): 1= dyspnea with strenuous activity, such as climbing three flights of stairs or heavy housework; $2=$ dyspnea with mild activity, such as climbing one flight of stairs or light housework; $3=$ dyspnea with minimal activity, such as walking 20 to 50 feet; and 4= dyspnea at rest or while eating or talking. Dyspnea scores for the evaluation of symptoms have been validated, as they correlate well with pulmonary function parameters (22).

\section{CT scanning protocol}

All images were obtained with one of three CT systems (uCT 780, United Imaging, Shanghai, China; Optima 660, GE Healthcare, Milwaukee, WI, USA; SOMATOM Definition $\mathrm{AS}_{+}$, Siemens Healthineers, Erlangen, Germany); patients were scanned in the supine position. The main scanning parameters were as follows: tube voltage, $120 \mathrm{kVp}$; automatic tube current modulation (ATCM), 30-70 mA s; pitch, 0.99-1.22 mm; matrix, 512 $\times 512$; field of view, $350 \mathrm{~mm} \times 350 \mathrm{~mm}$. All images were reconstructed with a slice thickness of $0.625-1.250 \mathrm{~mm}$ with the same increment. We analyzed peak CT images (CT image of the largest lesion range on multiple CT examinations during hospitalization) as well as CT images at discharge and during follow-up after discharge. Since physicians did not have a deep understanding of COVID-19 at the beginning of the epidemic and since the condition of patients in the acute phase changed rapidly, most patients received multiple CT scans during their hospitalization, with an average of $4.64 \pm 2.02$.

\section{Image interpretation}

The CT images were reviewed by two radiologists (15 and 10 years of experience in cardiothoracic imaging) who were blinded to the clinical classification results and dyspnea scores. Any discrepancies were resolved by discussion until a consensus was reached. The CT findings were described using internationally standard nomenclature defined by the Fleischner Society Glossary and peer-reviewed literature on viral pneumonia. The description terms included groundglass opacity (GGO; defined as hazy opacity that does not obscure the underlying pulmonary vessels and bronchi), crazy-paving pattern (defined as GGO with superimposed inter- and intralobular septal thickening), consolidation (defined as airspace opacity that obscures underlying parenchymal vessels and bronchi) and reticulation (defined as linear opacities) $(7,17,23)$. The main CT findings were defined as the most prominent of the four CT findings.

Data on the presence of bronchiolectasis, pleural effusion, enlarged mediastinal lymph nodes, pleural thickening and axial lesion distribution were also collected in the study. The lung lesion volume at different periods was assessed with 3D Slicer software (version 4.10.2, https:// www.slicer.org/). Image segmentation was performed manually by the above-mentioned two radiologists, with a minimum Hounsfield unit (HU) threshold of -750 and a maximum HU threshold of 80 (Figure 1). In addition, the residual lesion rate at discharge and at follow-up relative to the peak period was calculated according to the following formula: residual lesion rate $=$ discharge or follow-up CT 
lesion volume/peak CT lesion volume.

\section{Statistical analysis}

Statistical analyses were performed by SPSS (version 25; IBM, Chicago, IL, USA) and MedCalc version 19.1.3. Quantitative data are presented as the mean and standard deviation, and qualitative data are presented as counts and percentages of the total unless otherwise specified. Quantitative data were evaluated by $t$ test if they were normally distributed or MannWhitney U test otherwise. Qualitative data were compared between groups using Pearson's chi-square test or Fisher's exact test. Receiver operating characteristic (ROC) curves were constructed to analyze the abilities of clinical parameters and CT parameters to predict dyspnea. $\mathrm{P}<0.05$ was indicative of statistical significance.

\section{Results}

\section{Patient characteristics and clinical data}

A total of 337 survivors [mean age, $53.51 \pm 14.82$ years; $50.45 \%(170 / 337)$ men] were enrolled in this study; all of these patients underwent CT at the peak period, discharge, and follow-up except for 21 survivors who did not undergo CT at discharge. The time from onset to follow-up was $203.37 \pm 52.65$ days (102-361 days), and the time from discharge to follow-up was $164.13 \pm 52.04$ days (57-324 days).

None of the survivors had dyspnea before onset. At the time of follow-up CT, 91 survivors still had dyspnea, of which 7 had grade 2 dyspnea and the remainder had grade 1 dyspnea; thus, we classified dyspnea only by its presence or absence. The parameters with significant differences between patient groups are presented in Table 1. Patients with dyspnea after discharge were older and had more comorbidities, longer hospital stays, higher peak levels of CRP, LDH, D2-polymer, calcitonin, and neutrophil counts, and lower lymphocyte counts than survivors without dyspnea after discharge. The percentages of survivors who were male, required hormone administration, immunoglobulin injection, ICU admission, mechanical ventilation and classified as having severe or critical disease were significantly higher among those with dyspnea than among those without dyspnea. Survivors whose main CT finding was reticulations at follow-up had significantly longer hospital stays than those whose main CT finding was not reticulations $(33.07 \pm 14.40$ and $28.44 \pm 16.10$ days, respectively), with a $\mathrm{P}$ value of 0.003 . In $67.4 \%$ of the survivors, peak D2-polymer level was higher than normal during hospitalization.

\section{Evolution of CT findings}

On follow-up CT, $55.79 \%$ of the survivors still showed residual lesions; the residual lesion rate ranged from $1 \%$ to $83 \%$, and $15.43 \%$ of the survivors had a residual lesion rate exceeding $10 \%$. Lesion volume during the peak period, at discharge and at follow-up was $451.55 \pm 410.86 \mathrm{~cm}^{3}$, $298.27 \pm 365.06 \mathrm{~cm}^{3}$ and $54.17 \pm 162.30 \mathrm{~cm}^{3}$, respectively, indicating that lesion absorption occurred mainly after discharge (Figure 2).

Twenty-one survivors did not undergo CT at the time of discharge, and we excluded these patients from the comparison of images among the various timepoints. CT findings were compared between the peak period and discharge and between discharge and follow-up. The rates of most of the CT findings were significantly decreased at discharge and at follow-up; however, the rates of survivors with reticulations and reticulations as the main CT finding were significantly increased at discharge. Moreover, the rate of survivors with reticulations was significantly reduced at follow-up compared with discharge, but the rate of survivors with reticulations as the main CT finding was increased significantly at follow-up relative to discharge (Figure 3). Pleural effusion and enlarged mediastinal lymph nodes appeared only in the peak period, with 20 and 4 cases, respectively, so we did not include these two parameters in the analysis. The evolution patterns of the CT findings are presented in Table 2.

In addition, the differences in follow-up CT findings between less than 6 months $(121.38 \pm 31.20$ days $)$ after discharge and more than 6 months $(208.69 \pm 24.89$ days $)$ after discharge were analyzed (Table 3). The residual lesion rate and the rate of survivors with subpleural distribution of lung lesions were significantly reduced on follow-up CT more than 6 months after discharge compared with followup CT within 6 months after discharge.

\section{Comparison of CT findings in survivors with and without dyspnea}

CT findings were evaluated for significant differences between survivors with and without dyspnea. Survivors with dyspnea showed lower rates of GGO at the peak period and lower rates of subpleural distribution at the peak period and at discharge. Survivors with dyspnea had significantly 
Table 1 Comparison of patient characteristics and clinical data between survivors with and without dyspnea

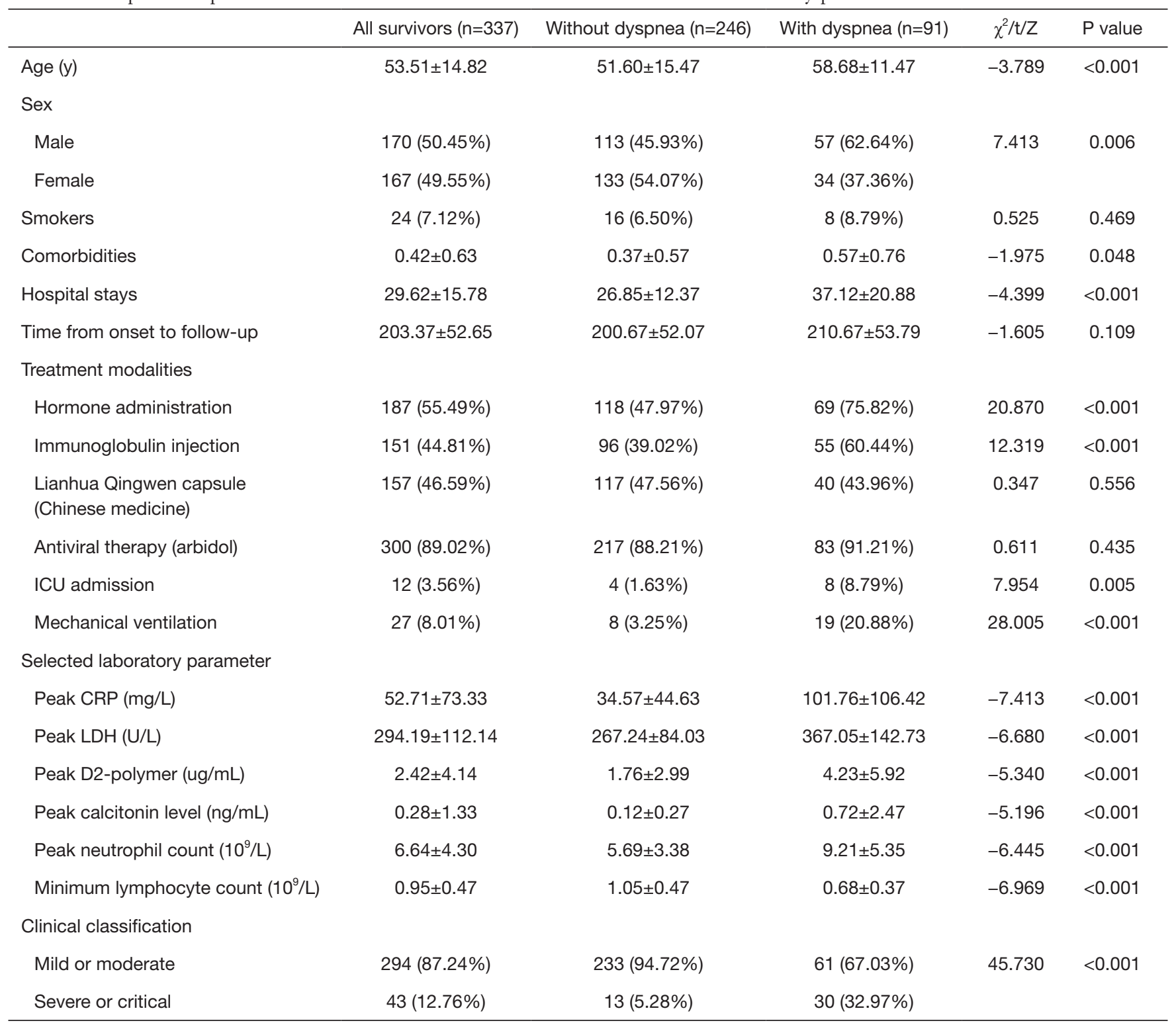

ICU, intensive care unit; CRP, C-reactive protein; LDH, lactate dehydrogenase.

large pulmonary vessel thrombi in COVID-19 (29,30). The observations that COVID-19 can cause an increase in D2-polymer and small vessel embolism has a limited effect on lung function may explain why no cases of pulmonary embolism were confirmed during hospitalization.

CT findings, including a crazy-paving pattern, consolidation, consolidation as the main CT finding, and lesion volume, were significantly reduced at discharge compared with during the peak period, reflecting lesion absorption. The above parameters, as well as GGOs and reticulations, were further significantly reduced during follow-up compared with at discharge. A previous study showed that only $9 \%$ of COVID-19 survivors were free of residual disease after three months, but another study showed that $57.7 \%$ of survivors had no lesions on followup CT after a three-month interval $(7,15)$. Our results showed that $44.21 \%$ of the survivors had no lesions at follow-up and that survivors with residual lesion rates greater than $10 \%$ accounted for only $15.43 \%$ of the study population, indicating that most of the lesions had been 

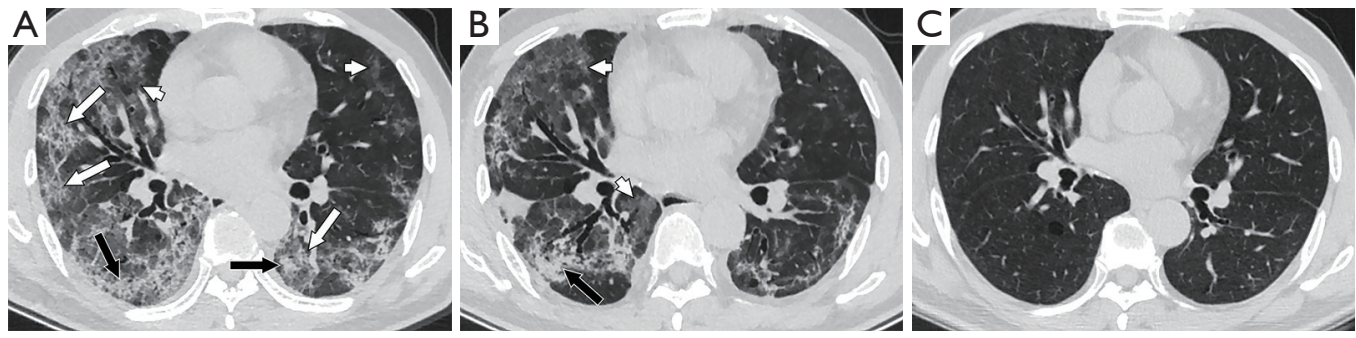

Figure 2 Thin-slice CT scan of a 48-year-old man with COVID-19 with dyspnea after discharge (A-C obtained at 35, 66 and 284 days after onset, respectively). Lesion volume was largest in the peak period, mainly manifested as crazy-paving patterns (white arrows), with some GGOs (white arrowheads) and a small amount of consolidations (black arrows) (A). At discharge, the lesion range was reduced, most of the crazy-paving patterns had disappeared, and the lesions mainly presented as GGOs (white arrowheads) and consolidations (black arrow) (B). In the follow-up CT at 284 days after discharge, almost all the lesions had been absorbed (C). CT, computed tomography; COVID-19, coronavirus disease 2019; GGOs, ground-glass opacities.
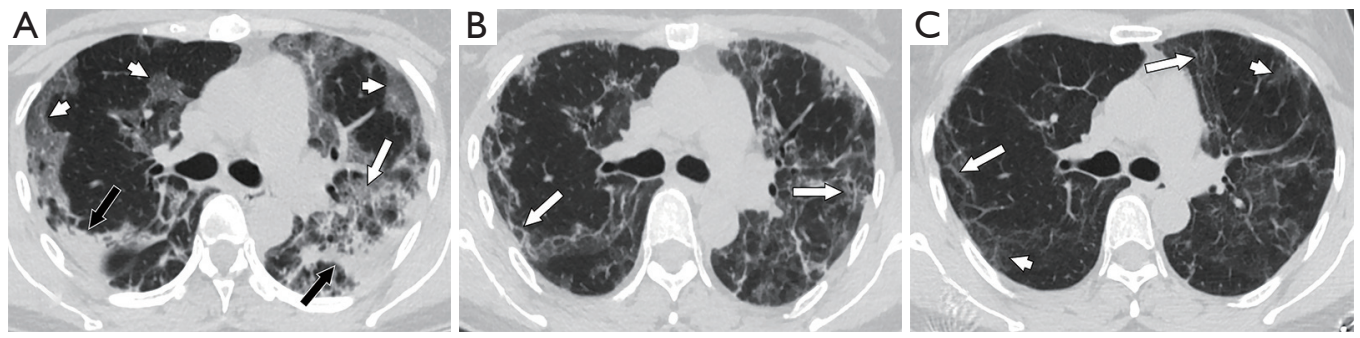

Figure 3 Thin-slice CT scan of a 40-year-old woman with COVID-19 with dyspnea after discharge (A-C obtained at 23, 70 and 254 days after onset, respectively). In the peak period, consolidation (black arrows), GGOs (white arrowheads) and crazy-paving patterns (white arrow) were observed bilaterally (A). At discharge, most areas of consolidation and the crazy-paving pattern had been absorbed, but reticulations (white arrows) had increased significantly (B). Only reticulations (white arrows) and small areas of ground-glass opacities (GGOs, white arrowheads) remained at follow-up (C). CT, computed tomography; COVID-19, coronavirus disease 2019; GGOs, ground-glass opacities.

higher rates or values of the following CT findings: (I) peak period: consolidation, bronchiolectasis, random or diffuse distribution, pleural thickening, and lesion volume; (II) at discharge: crazy-paving pattern, reticulations, reticulation as the main CT findings, bronchiolectasis, random or diffuse distribution, pleural thickening, lesion volume and residual lesion rate; and (III) follow-up: GGOs, reticulations, GGOs as the main CT finding, reticulations as the main CT findings, bronchiolectasis, random or diffuse distribution, pleural thickening, lesion volume, and residual lesion rate. A comparison of CT findings between survivors with and without dyspnea is shown in Table 4.

\section{ROC analysis}

Figure 4 and Table 5 show the clinical parameters and CT findings with the top 10 largest areas under the curve
(AUCs) that were used to identify dyspnea in survivors. Lesion volume at discharge had the largest AUC (0.820) and the highest sensitivity (0.908), and GGOs at followup had the highest specificity (0.799). The presence of GGOs and the presence of reticulations were analyzed as qualitative data, so cutoff values could not be obtained. The parameters of the top 10 AUCs were pairwise compared, and most of the comparisons between the first 5 parameters and the last 5 parameters showed significant differences.

\section{Discussion}

A total of $27.00 \%$ of the survivors had dyspnea at follow-up; this rate is slightly less than the rate of one-third observed among patients with severe acute respiratory syndrome (SARS) and similar to the finding of pulmonary function tests in COVID-19 survivors at 3 months after discharge, 
Table 2 Evolution of CT findings

\begin{tabular}{|c|c|c|c|c|c|}
\hline CT findings & Peak period $(n=316)$ & \multicolumn{2}{|c|}{ Discharge $(n=316)$} & \multicolumn{2}{|c|}{ Follow-up $(n=316)$} \\
\hline GGO, n (\%) & $281(88.92 \%)$ & 263 (83.23\%) & 0.039 & 106 (33.54\%) & $<0.001$ \\
\hline Crazy-paving pattern & $179(56.65 \%)$ & $128(40.51 \%)$ & $<0.001$ & $24(7.59 \%)$ & $<0.001$ \\
\hline Reticulation & 226 (71.52\%) & $256(81.01 \%)$ & 0.005 & $146(46.20 \%)$ & $<0.001$ \\
\hline \multicolumn{6}{|l|}{ Main CT finding } \\
\hline GGO & $163(51.58 \%)$ & $160(50.63 \%)$ & 0.811 & $53(16.77 \%)$ & $<0.001$ \\
\hline Crazy-paving pattern & $58(18.35 \%)$ & $53(16.77 \%)$ & 0.601 & $11(3.48 \%)$ & $<0.001$ \\
\hline Consolidation & $84(26.58 \%)$ & $19(6.01 \%)$ & $<0.001$ & $8(2.53 \%)$ & 0.030 \\
\hline \multicolumn{6}{|l|}{ Distribution of lesions } \\
\hline Subpleural & $190(60.13 \%)$ & 202 (63.92\%) & 0.325 & 139 (43.99\%) & $<0.001$ \\
\hline Random & $59(18.67 \%)$ & $60(18.99 \%)$ & 0.919 & $17(5.38 \%)$ & $<0.001$ \\
\hline Diffuse & $67(21.20 \%)$ & $42(13.29 \%)$ & 0.008 & $11(3.48 \%)$ & $<0.001$ \\
\hline Pleural thickening & $70(22.15 \%)$ & $50(15.82 \%)$ & 0.043 & $11(3.48 \%)$ & $<0.001$ \\
\hline Lesion volume $\left(\mathrm{cm}^{3}\right)$ & $451.55 \pm 410.86$ & $298.27 \pm 365.06$ & $<0.001$ & $54.17 \pm 162.30$ & $<0.001$ \\
\hline
\end{tabular}

*, the $\mathrm{P}$ value represents the comparison between discharge and peak period; ${ }^{*}$, the $\mathrm{P}$ value represents the comparison between follow-up and discharge. CT, computed tomography; GGO, ground-glass opacity.

showing residual pulmonary function abnormalities in $25.4 \%$ of patients $(24,25)$.

Survivors of COVID-19 with dyspnea after discharge were older and had more comorbidities than those without dyspnea after discharge, and male survivors were more likely than female survivors to have dyspnea after discharge. Previous SARS studies have shown that men and older survivors are more likely to develop fibrosis; similarly, our study shows that survivors with dyspnea have more reticulations, which may be the cause of dyspnea (26). Parameters that reflect disease severity, such as the duration of hospital stay, hormone administration, ICU admission, and clinical classification, showed significant differences between survivors with and without dyspnea, suggesting that more severe illness during hospitalization was associated with a higher risk of dyspnea. In addition, the hospital stays were significantly longer among survivors whose main CT finding during follow-up was reticulation, suggesting that the more serious the disease was, the longer hospital stay was, the more reticulation formed after discharge, and the more likely it was that dyspnea occurred.
Peak CRP, peak LDH, peak D2-polymer, peak calcitonin, peak neutrophil count, and minimum lymphocyte count are laboratory parameters indicative of disease severity, inflammatory response, and tissue damage in the acute phase (27). These parameters showed significant differences between survivors with and without dyspnea, similar to results obtained in SARS survivors $(22,24)$. D2-polymer is frequently elevated in acute venous thromboembolism (VTE) but is nonspecific, being frequently elevated in many other nonthrombotic conditions, including pregnancy, cancer and inflammation, and it has been shown to be frequently elevated in COVID-19-positive patients in the absence of VTE (28). In our study, $67.4 \%$ of the survivors had an elevated peak D2-polymer level during hospitalization, but no cases of pulmonary embolism were confirmed during hospitalization, and only 3 patients were readmitted for pulmonary embolism within 5-7 months after discharge. Since COVID-19 could not be identified as the cause of pulmonary embolism in these patients, we excluded these cases. Evidence suggests that small vessel pulmonary thrombi are more commonly involved than 
Table 3 Comparisons of follow-up CT findings between survivors at less than 4 months and more than 4 months after discharge

\begin{tabular}{|c|c|c|c|}
\hline Follow-up CT findings & Within 6 months after discharge $(n=172)$ & More than 6 months after discharge $(n=165)$ & $P$ \\
\hline Crazy-paving pattern & $16(9.30 \%)$ & $12(7.27 \%)$ & 0.500 \\
\hline Reticulation & $83(48.26 \%)$ & $76(46.06 \%)$ & 0.687 \\
\hline Consolidation & $4(2.33 \%)$ & $8(4.85 \%)$ & 0.212 \\
\hline GGO & $34(19.77 \%)$ & $23(13.94 \%)$ & 0.154 \\
\hline Crazy-paving pattern & $5(2.91 \%)$ & $6(3.64 \%)$ & 0.706 \\
\hline Consolidation & $4(2.33 \%)$ & $6(3.64 \%)$ & 0.698 \\
\hline Reticulation & $56(13.37 \%)$ & $42(25.45 \%)$ & 0.151 \\
\hline Subpleural & $84(48.84 \%)$ & $60(36.36 \%)$ & 0.021 \\
\hline Random & $8(4.65 \%)$ & $9(5.45 \%)$ & 0.736 \\
\hline Diffuse & $7(4.07 \%)$ & $8(4.85 \%)$ & 0.729 \\
\hline Pleural thickening & $6(3.49 \%)$ & $5(3.03 \%)$ & 0.813 \\
\hline Lung lesion volume $\left(\mathrm{cm}^{3}\right)$ & $57.38 \pm 165.11$ & $50.63 \pm 150.81$ & 0.102 \\
\hline Residual lesion rate (\%) & $7.80 \pm 15.43$ & $5.81 \pm 13.20$ & 0.026 \\
\hline
\end{tabular}

CT, computed tomography; GGO, ground-glass opacity.

absorbed by 3 months after disease onset. Among the main CT findings, reticulations were significantly increased at discharge and further significantly increased at follow-up. We found that the rate of survivors with reticulations was significantly reduced at follow-up, whereas that of survivors with reticulation as the main CT finding was significantly increased at follow-up. This difference may be due to the fact that other lesions are relatively easy to absorb, thus leaving only reticulations; alternatively, it may be that reticulations are representative of lesions in the late stage in some survivors. Previous short-term follow-up studies have suggested that reticulations are indicative of fibrosis (31-33). However, our results showed that nearly half of the reticulations observed on CT at discharge were absorbed during follow-up, suggesting that not all reticulations represent true fibrosis. Approximately one-quarter of survivors had bronchiolectasis during the peak period and at discharge; the frequency significantly decreased to approximately one in eight at follow-up. We observed that bronchiolectasis was most obvious at discharge and then recovered significantly during follow-up as lesion volume significantly reduced. A small number of patients showed pleural effusion and enlarged mediastinal lymph nodes, consistent with previous reports (34). Table 3 shows that among the CT parameters, only the residual lesion rate and rate of subpleural distribution were significantly decreased at more than 6 months after discharge compared with less than 6 months after discharge. This indicates that the lesions were still being absorbed more than 6 months after discharge. Moreover, GGOs continued to be observed on CT images in $30 \%$ of the survivors more than 6 months after discharge; these lesions may be absorbed over time.

The rates of reticulation as the main CT finding at discharge and at follow-up were significantly higher among survivors with dyspnea than survivors without dyspnea, which is consistent with long-term follow-up results in SARS patients $(22,26)$. These results may have been obtained because survivors with dyspnea have larger lesions that are incompletely absorbed at discharge and at follow-up, and chest CT showed more reticulations in these survivors. The rate of bronchiolectasis in survivors with dyspnea was significantly higher than that in survivors without dyspnea at 


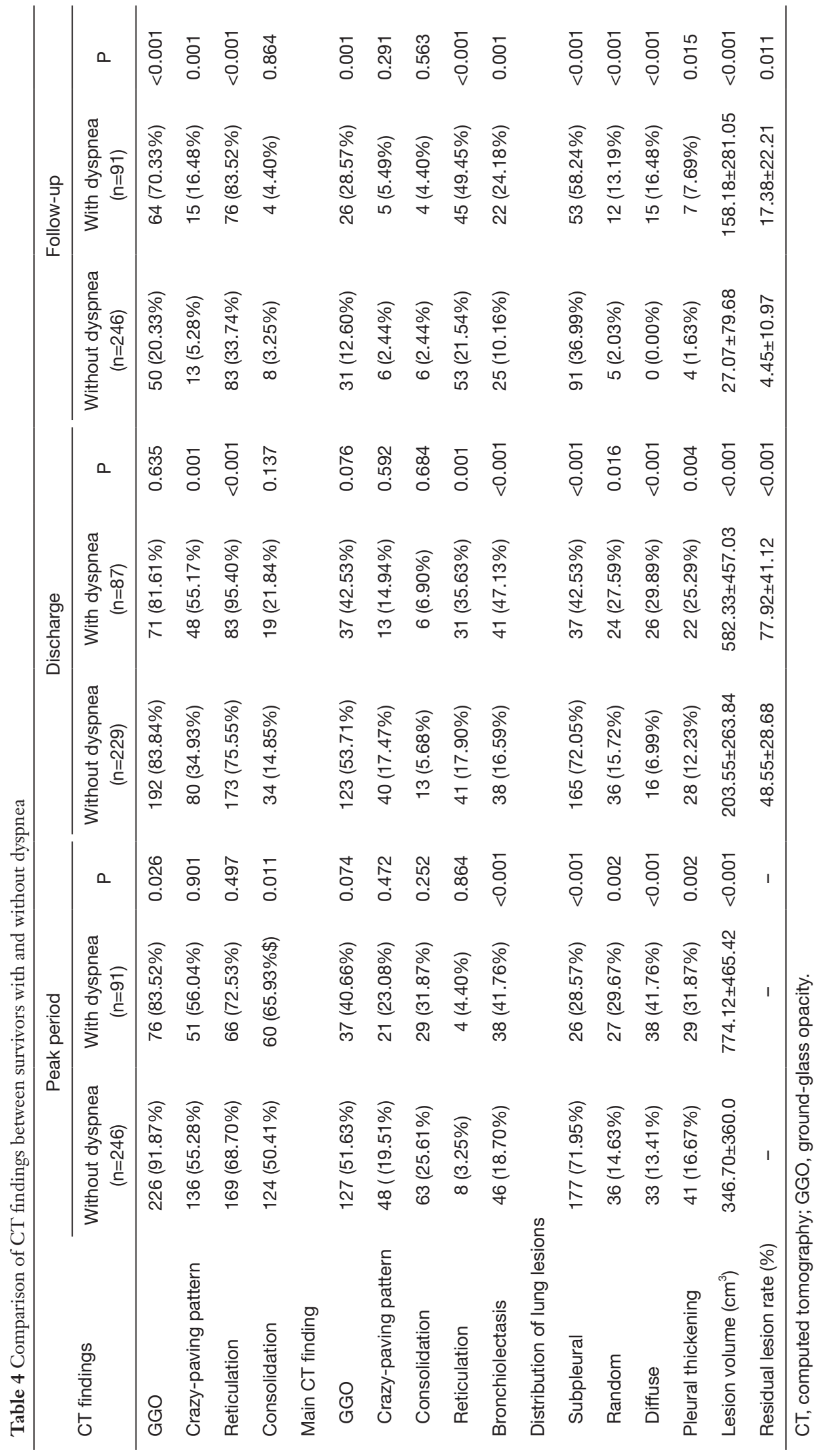




\section{Page 10 of 12}

the three time points. We found that bronchiolectasis tended to occur in patients with larger lesion volumes. Since patients with dyspnea had significantly larger lesion volumes than those without dyspnea, the incidence of bronchiolectasis was higher in the former. At follow-up, the rates of GGOs and reticulations were higher in survivors with dyspnea than in those without dyspnea. This result may be due to the fact that survivors with dyspnea have larger lesions and a higher residual lesion rate, with these incompletely absorbed lesions

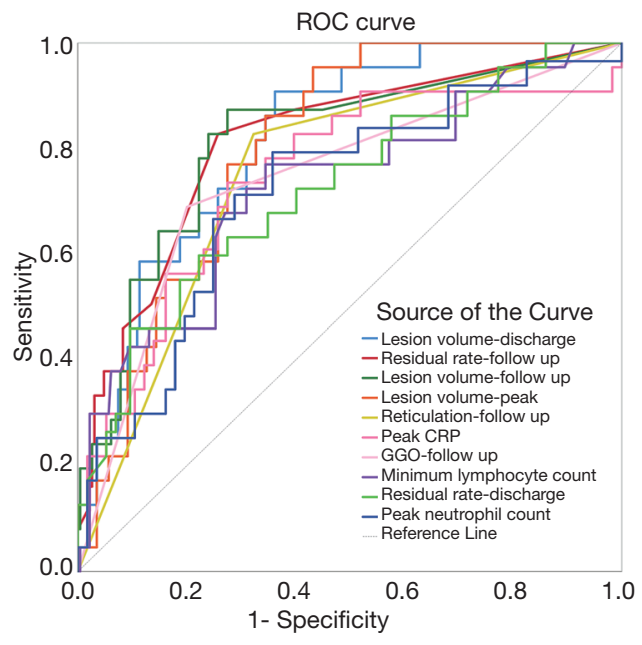

Figure 4 ROC curves for the top 10 clinical parameters and CT findings for differentiating between survivors with and without dyspnea. ROC, receiver operator characteristic; CT, computed tomography; CRP, C-reactive protein; GGO, ground-glass opacity.
Yin et al. Long-term chest CT follow-up in COVID-19 survivors

manifesting as GGOs and reticulations.

As shown in Table 5 and Figure 4, among the parameters for identifying dyspnea, most of the top 10 parameters with the largest AUC were related to the lesion volume. The paired comparison results showed that the AUC values of the first 5 volume-related parameters were significantly larger than those of the last 5 parameters, which indicated that the parameters associated with lesion volume are the most accurate predictors of postdischarge dyspnea in COVID-19 survivors. The AUC for lesion volume at discharge was the largest (0.820), suggesting that the larger the lesion is at discharge, the more likely the persistence of dyspnea is after discharge.

This study has several limitations. First, it was a singlecenter retrospective study. However, there have been few long-term follow-up CT studies of COVID-19, and expert opinions on practice guidelines and rehabilitation need to be validated in sufficiently powered longitudinal studies. Second, we did not have data on the pulmonary function of survivors, but a previous study has validated dyspnea for the evaluation of symptoms based on its strong correlation with pulmonary function parameters (21). Third, some GGOs and reticulations were not completely absorbed according to the follow-up CT images; therefore, a longer time is required to observe the complete process. Fourth, the proportion of patients from the ICU setting in our sample was low. However, according to a report from $N$ Engl $7 \mathrm{Med}$, only a small number of confirmed COVID-19 pneumonia patients $(5.0 \%)$ were admitted to the ICU (2).

Table 5 Area under the curve

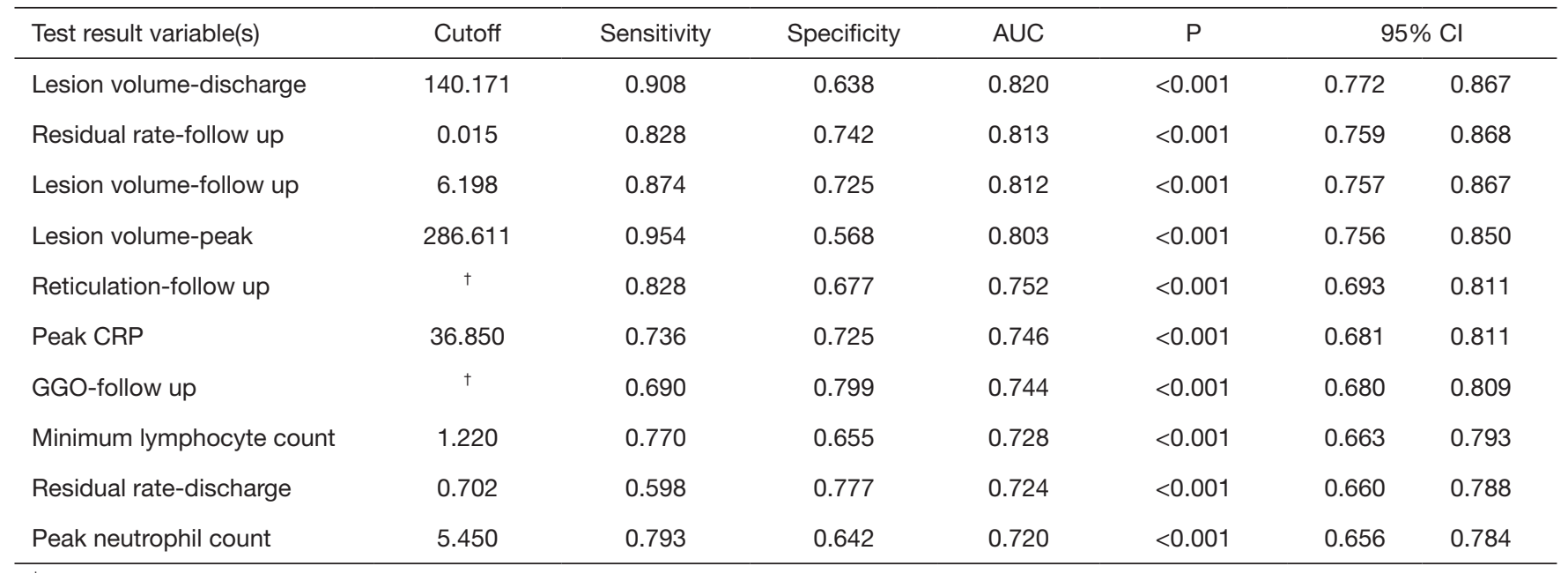

${ }^{\dagger}$, the dichotomous variable is considered to have this CT finding as the threshold. AUC, area under the curve; CRP, C-reactive protein; GGO, ground-glass opacity. 


\section{Conclusions}

In this longitudinal study of COVID-19 patients during the peak period, at discharge, and at follow-up, the lesion volume in survivors decreased gradually over time, especially after discharge. Among the CT findings and clinical parameters, CT findings associated lesion volume were the best predictors of dyspnea in COVID-19 survivors. In the long-term management of discharged COVID-19 survivors, extra vigilance is required regarding the persistence of dyspnea when the lesion volume remains large at discharge. Such patients need active rehabilitation after discharge to improve their long-term quality of life.

\section{Acknowledgments}

Funding: This work was supported by the Hubei Provincial Novel Pneumonia Emergency Science and Technology Project [grant No. 2020FCA021] to [LX]; and the Huazhong University of Science and Technology Novel Coronavirus Pneumonia Emergency Science and Technology Project [grant No. 2020kfyXGYJ014] to [LX].

\section{Footnote}

Reporting Checklist: The authors have completed the STROBE reporting checklist. Available at https://dx.doi. org/10.21037/atm-21-1438

Data Sharing Statement: Available at https://dx.doi. org/10.21037/atm-21-1438

Conflicts of Interest: All authors have completed the ICMJE uniform disclosure form (available at https://dx.doi. org/10.21037/atm-21-1438). LX reports that he was supported by the Hubei Provincial Novel Pneumonia Emergency Science and Technology Project [grant No. 2020FCA021]; and the Huazhong University of Science and Technology Novel Coronavirus Pneumonia Emergency Science and Technology Project [grant No. 2020kfyXGYJ014]. The other authors have no conflicts of interest to declare.

Etbical Statement: The authors are accountable for all aspects of the work in ensuring that questions related to the accuracy or integrity of any part of the work are appropriately investigated and resolved. The study was conducted in accordance with the Declaration of
Helsinki (as revised in 2013). The study was approved by ethics committee of Wuhan Tongji Hospital (No. TJIRB20210120), and individual consent for this retrospective analysis was waived.

Open Access Statement: This is an Open Access article distributed in accordance with the Creative Commons Attribution-NonCommercial-NoDerivs 4.0 International License (CC BY-NC-ND 4.0), which permits the noncommercial replication and distribution of the article with the strict proviso that no changes or edits are made and the original work is properly cited (including links to both the formal publication through the relevant DOI and the license). See: https://creativecommons.org/licenses/by-nc-nd/4.0/.

\section{References}

1. Weekly operational update on COVID-19-22 March 2021. Accessed March 22, 2021. Available online: https:// www.who.int/publications/m/item/weekly-operationalupdate-on-covid-19---22-march-2021

2. Guan WJ, Ni ZY, Hu Y, et al. Clinical Characteristics of Coronavirus Disease 2019 in China. N Engl J Med 2020;382:1708-20.

3. Liu C, Ye L, Xia R, et al. Chest Computed Tomography and Clinical Follow-Up of Discharged Patients with COVID-19 in Wenzhou City, Zhejiang, China. Ann Am Thorac Soc 2020;17:1231-7.

4. Chen HJ, Qiu J, Wu B, et al. Early chest CT features of patients with 2019 novel coronavirus (COVID-19) pneumonia: relationship to diagnosis and prognosis. Eur Radiol 2020;30:6178-85.

5. Fu Z, Tang N, Chen Y, et al. CT features of COVID-19 patients with two consecutive negative RT-PCR tests after treatment. Sci Rep 2020;10:11548.

6. Yu M, Liu Y, Xu D, et al. Prediction of the Development of Pulmonary Fibrosis Using Serial Thin-Section CT and Clinical Features in Patients Discharged after Treatment for COVID-19 Pneumonia. Korean J Radiol 2020;21:746-55.

7. Salehi S, Abedi A, Balakrishnan S, et al. Coronavirus disease 2019 (COVID-19) imaging reporting and data system (COVID-RADS) and common lexicon: a proposal based on the imaging data of 37 studies. Eur Radiol 2020;30:4930-42.

8. Prescott HC, Girard TD. Recovery From Severe COVID-19: Leveraging the Lessons of Survival From Sepsis. JAMA 2020;324:739-40.

9. Shaw B, Daskareh M, Gholamrezanezhad A. The lingering manifestations of COVID-19 during and 
after convalescence: update on long-term pulmonary consequences of coronavirus disease 2019 (COVID-19). Radiol Med 2021;126:40-6.

10. Gandhi D, Ahuja K, Grover H, et al. Review of X-ray and computed tomography scan findings with a promising role of point of care ultrasound in COVID-19 pandemic. World J Radiol 2020;12:195-203.

11. Akl EA, Blažić I, Yaacoub S, et al. Use of Chest Imaging in the Diagnosis and Management of COVID-19: A WHO Rapid Advice Guide. Radiology 2021;298:E63-9.

12. Yin X, Min X, Nan Y, et al. Assessment of the Severity of Coronavirus Disease: Quantitative Computed Tomography Parameters versus Semiquantitative Visual Score. Korean J Radiol 2020;21:998-1006.

13. Wei J, Yang H, Lei P, et al. Analysis of thin-section CT in patients with coronavirus disease (COVID-19) after hospital discharge. J Xray Sci Technol 2020;28:383-9.

14. Shah AS, Wong AW, Hague CJ, et al. A prospective study of 12 -week respiratory outcomes in COVID-19-related hospitalisations. Thorax 2021;76:402-4.

15. van den Borst B, Peters JB, Brink M, et al. Comprehensive health assessment three months after recovery from acute COVID-19. Clin Infect Dis 2020. [Epub ahead of print]. doi: 10.1093/cid/ciaa1750.

16. Tabatabaei SMH, Rajebi H, Moghaddas F, et al. Chest CT in COVID-19 pneumonia: what are the findings in midterm follow-up? Emerg Radiol 2020;27:711-9.

17. Gandhi D, Jain N, Khanna K, et al. Current role of imaging in COVID-19 infection with recent recommendations of point of care ultrasound in the contagion: a narrative review. Ann Transl Med 2020;8:1094.

18. Huang C, Huang L, Wang Y, et al. 6-month consequences of COVID-19 in patients discharged from hospital: a cohort study. Lancet 2021;397:220-32.

19. Han X, Fan Y, Alwalid O, et al. Six-month Follow-up Chest CT Findings after Severe COVID-19 Pneumonia. Radiology 2021;299:E177-86.

20. National Health Commission of the People's Republic of China. Diagnosis and Treatment Protocol for Novel Coronavirus Pneumonia (Trial Version 7). Accessed March 4, 2020. Available online: http://www.nhc.gov.cn/xcs/zhen gcwj/202003/46c9294a7dfe4cef80dc7f5912eb1989/files/ce 3e6945832a438eaae415350a8ce964.pdf

21. Staples CA, Müller NL, Vedal S, et al. Usual interstitial pneumonia: correlation of CT with clinical, functional, and radiologic findings. Radiology 1987;162:377-81.

22. Hsu HH, Tzao C, Wu CP, et al. Correlation of high-resolution CT, symptoms, and pulmonary function in patients during recovery from severe acute respiratory syndrome. Chest 2004;126:149-58.

23. Hansell DM, Bankier AA, MacMahon H, et al. Fleischner Society: glossary of terms for thoracic imaging. Radiology 2008;246:697-722.

24. Chan KS, Zheng JP, Mok YW, et al. SARS: prognosis, outcome and sequelae. Respirology 2003;8 Suppl:S36-40.

25. Zhao YM, Shang YM, Song WB, et al. Follow-up study of the pulmonary function and related physiological characteristics of COVID-19 survivors three months after recovery. EClinicalMedicine 2020;25:100463.

26. Antonio GE, Wong KT, Hui DS, et al. Thin-section $\mathrm{CT}$ in patients with severe acute respiratory syndrome following hospital discharge: preliminary experience. Radiology 2003;228:810-5.

27. Xu L, Mao Y, Chen G. Risk factors for 2019 novel coronavirus disease (COVID-19) patients progressing to critical illness: a systematic review and meta-analysis. Aging (Albany NY) 2020;12:12410-21.

28. Patel L, Gandhi D, Westergard E, et al. COVID-19 and venous thromboembolism: Known and unknown for imaging decisions. World J Radiol 2021;13:64-74.

29. Ooi MWX, Rajai A, Patel R, et al. Pulmonary thromboembolic disease in COVID-19 patients on CT pulmonary angiography - Prevalence, pattern of disease and relationship to D-dimer. Eur J Radiol 2020;132:109336.

30. Alonso-Fernández A, Toledo-Pons N, Cosío BG, et al. Prevalence of pulmonary embolism in patients with COVID-19 pneumonia and high D-dimer values: A prospective study. PLoS One 2020;15:e0238216.

31. Liu R, Lei C, Liao X, et al. Semi-quantitative analysis for the dynamic chest CT imaging features from onset to recovery in severe and critical COVID-19. Radiol Infect Dis 2020;7:114-22.

32. Zhou Y, Zheng Y, Yang Q, et al. Cohort study of chest CT and clinical changes in 29 patients with coronavirus disease 2019 (COVID-19). Eur Radiol 2020;30:6213-20.

33. Liang T, Liu Z, Wu CC, et al. Evolution of CT findings in patients with mild COVID-19 pneumonia. Eur Radiol 2020;30:4865-73.

34. Chung M, Bernheim A, Mei X, et al. CT Imaging Features of 2019 Novel Coronavirus (2019-nCoV). Radiology 2020;295:202-7.

Cite this article as: Yin $\mathrm{X}, \mathrm{Xi} \mathrm{X}$, Min $\mathrm{X}$, Feng Z, Li B, Cai W, Fan C, Wang L, Xia L. Long-term chest CT follow-up in COVID-19 Survivors: 102-361 days after onset. Ann Transl Med 2021;9(15):1231. doi: 10.21037/atm-21-1438 